\title{
On Surface Gravity Wave Energies
}

\author{
Kern E. Kenyon
}

4632 North Lane, Del Mar, USA

Correspondence to: Kern E. Kenyon, kernken@aol.com

Keywords: Surface Gravity Waves, Energy

Received: August 26, 2020 Accepted: October 17, 2020 Published: October 20, 2020

Copyright $\odot 2020$ by author(s) and Scientific Research Publishing Inc.

This work is licensed under the Creative Commons Attribution International License (CC BY 4.0).

http://creativecommons.org/licenses/by/4.0/

\section{(c) (i) Open Access}

\section{ABSTRACT}

Kinetic and potential energies of a propagating surface gravity wave are calculated for the orbiting fluid particles. These energies are then compared with the respective standard theoretical quantities. Based on earlier work, it is concluded that the two sets of energies will not agree. To substantiate the prediction observations are needed, or a new theory for the depth decay rate of the particle orbits would work. Considerably less algebraic effort is involved in the present analysis than what was done in the past.

\section{INTRODUCTION}

Surface gravity waves possess kinetic (KE) and potential (PE) energies, as presented in many fluid dynamics textbooks. Two references are given here $[1,2]$, but the demonstrations in the others are all very similar. What may be confusing to some readers is that treatments of KE are very different from those of $\mathrm{PE}$, and it is not clear why that should be so. To criticize the standard derivations of KE and PE and to offer a different approach to calculating them are the objectives of this essay.

Calculating the total potential energy per unit horizontal area of a propagating surface gravity wave would seem to have acquired no complaints: the work done against gravity to change a level air/water boundary into a sinusoidal shape. What could be wrong with that? For a static situation, there is no quarrel with this method. However, the problem is not static, because fluid moves along streamlines that have curves in the steady state picture, where the fluid flows by the observer but the wave profile does not move. Fluid particles experience a centrifugal force when traveling along a curved path. In other words, gravity is not the only force in the problem that work can be done against.

Also at any given mean depth, there is no way to calculate what the PE is by the traditional technique. It makes no sense even to ask the question. This is in sharp contrast to the KE which can be computed at any mean depth. One way out of this strange dilemma, apparently not thought of by theoreticians historically, is to apply Bernoulli's law to streamlines other than just the surface one. Bernoulli's law is an energy statement containing $\mathrm{KE}$ and $\mathrm{PE}$ terms.

Computing the KE of surface gravity waves requires the velocity of the moving fluid. No other way to start on this objective has been found classically except to adopt the assumption of irrotational motion, or 
potential flow, originally brought in from physics. The PE calculation, on the other hand, is independent of the irrotational assumption. Also the nonlinearity of the fluid dynamics (Navier-Stokes) equations is dealt with by applying perturbation expansions (from astronomy). Again the PE result does not require such an operation.

\section{METHOD}

In the fixed reference frame, fixed to the solid bottom of a lake or ocean, surface gravity waves pass by an observer who could notice the orbital motion of neutrally buoyant particles, which has been done in the lab. several times. In fact, all fluid particles within the depth of wave influence have circular orbits. For the present purpose the circles are considered to be closed. Therefore, kinetic energy exists throughout the water column containing orbiting fluid particles.

$$
\mathrm{KE}=\frac{1}{2} \rho V^{2}
$$

where $V$ is the speed of the particle tangent to the orbit.

Each orbiting particle experiences an outward centrifugal force. Since turbulence or chaos do not occur, the centrifugal force must be balanced by an equal but opposite (inward) force, and it has the form of a spring force. Consequently, each orbiting particle has potential energy also.

$$
\mathrm{PE}=\frac{1}{2} \rho\left(\frac{V^{2}}{r}\right) r
$$

And $r$ is the orbital radius, which is a function of the depth $z$.

Equations (1) and (2) are equal at each mean depth below the surface, putting KE and PE on a more common footing, so to speak, using no perturbations and not making the irrotational assumption.

Also

$$
V=\omega r(z)
$$

where $\omega$ is the constant wave frequency.

\section{DISCUSSION}

To my knowledge neither of the classical formulas for the KE and PE of the propagating surface gravity wave has ever been held up to observations for comparisons. Now comes a different theoretical calculation of those quantities that in all probability will disagree with the existing ones.

Take the standard KE

$$
\mathrm{KE}=\frac{1}{2} \rho\left(u^{2}+w^{2}\right)
$$

where $u$ and $w$ are the horizontal and vertical components of the fluid velocity. When the potential flow solution for $u$ and $w$ are inserted into (4), the result is

$$
\mathrm{KE}=\frac{1}{2} \rho(a \omega)^{2} \mathrm{e}^{2 k z}
$$

where $a$ is the wave amplitude, $k$ is the wave number and the total depth of water is assumed to be much greater than the wavelength (deep water waves).

Putting (3) into (1) gives

$$
\mathrm{KE}=\frac{1}{2} \rho \omega^{2} r^{2}(z)
$$

And the only way (6) can agree with (5) is if the orbital radius of the particles decreases exponentially 
with increasing depth with the e-folding scale equal to the wave number. Odds are this will not happen as predicted by earlier work [3]. Needed for proof one way or the other is $r(z)$ obtained either from observations of sufficiently high quality or another theory. Meanwhile a major flaw in the reasoning behind the application of potential flow to surface gravity waves has been pointed out [4].

Gravity wave energy, based on the orbital motion of the fluid particles, does not turn out to be explicitly dependent on gravity, in the most convenient way to obtain the energy expressions. In none of the above equations does the acceleration of gravity appear. Gravity is not too far away though, because the classical dispersion relation $\left(\omega^{2}=g k\right)$, also not making an appearance here, can be entered into the equations at any time.

\section{CONCLUSION}

A most important property of the surface gravity wave, its energy, may not be what classical scholars have produced. Working up the potential and kinetic energies of propagating waves from the point of view of the orbital motion of the fluid particles, which apparently has not occurred to any scientist before to carry out, gives a result that will most likely be different when future work is done, either by a new theory or by careful measurements, based on a previous prediction.

\section{CONFLICTS OF INTEREST}

The authors declare no conflicts of interest regarding the publication of this paper.

\section{REFERENCES}

1. Proudman, J. (1953) Dynamical Oceanography. John Wiley, New York, 377-380.

2. Kundu, P.K. (1990) Fluid Mechanics. Academic Press, San Diego, 198-200.

3. Kenyon, K.E. (2013) Depth Decay Rate for Surface Gravity Wave Pressure and Velocity. Natural Science, 5, No. 8.

4. Kenyon, K.E. (2020) Frictionless Surface Gravity Waves. Natural Science, 12, 199-201.

https://doi.org/10.4236/ns.2020.124017 